\title{
高性能聚集诱导发光纳米探针用于肿瘤切除手术导航
}

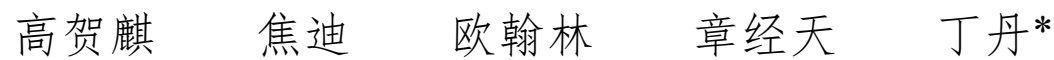 \\ (南开大学生命科学学院 药物化学生物学国家重点实验室 生物活性材料教育部重点实验室 天津 300071)
}

\begin{abstract}
摘要 远红/近红外(FR/NIR)发射荧光材料因其在生物成像领域的巨大应用潜力而受到广泛关注. 然而, 根据 “能隙” 规则, 电子带隙减小时, 非辐射失活途径占据能量耗散主导地位, 因此制备高量子产率、长发射波长的有机 FR/NIR 材 料仍然是一个挑战. 本文通过引入扭曲构象基团(四苯基乙烯)对分子聚集态进行调控，合成了一系列基于苯并硒二唑 的供体-受体-供体(D-A-D)型化合物. 化合物 TPE-DPA-Se 在纳米粒子状态下表现出明亮的苂光发射, 其量子产率可高 达 $16.48 \%$, 证明其在水体系中仍具有良好的发射性能, 适用于多个领域. 本研究进一步证实了其优秀的细胞成像能力, 并将其成功地应用于荧光成像引导的手术导航, 特别是微小转移瘤的术中检测.
\end{abstract}

关键词 聚集诱导发光; 远红/近红外; 纳米粒子; 细胞成像; 手术导航

\section{High Performance Aggregation-Induced Emission Nanoprobes for Image-Guided Cancer Surgery}

\author{
Heqi Gao Di Jiao Hanlin Ou Jingtian Zhang Dan Ding* \\ (State Key Laboratory of Medicinal Chemical Biology, Key Laboratory of Bioactive Materials, Ministry of Education, \\ College of Life Sciences, Nankai University, Tianjin 300071, China)
}

\begin{abstract}
Far red/near infrared (FR/NIR) materials have attracted wide attention due to their great potential in various applications, particularly in bio-imaging. However, it is still a challenge to manufacture organic FR/NIR materials with quite high efficiency and long emission wavelength, owing to the dominance of non-radiative deactivation in the dissipation of absorbed light excitation energy when the electronic bandgap decreases. Herein, a series of donor-acceptor-donor (D-A-D) type compounds based on benzoselenidazole are developed through the regulation of molecular aggregation states by twisted conformation groups. In one hand, the compound TPE-DPA-Se (tetraphenylethylene-diphenylamine-benzoselenidazole) exhibits the best aggregation-induced emission (AIE) properties among these compounds. In another hand, the obtained TPE-DPA-Se showed over $150 \mathrm{~nm}$ Stokes shift, which can be used to avoid the interference between excitation and emission light, as well as the near-infrared emission spectrum away from the organism auto-fluorescence, which was beneficial for the bio-application. Next, density functional theory (DFT) calculation was carried out with Gaussian $09 \mathrm{~W}$ program at B3LYP/6-31G** level to determine the highest occupied molecular orbital (HOMO), the lowest unoccupied molecular orbital (LUMO) distributions and the optimized structures of these compounds. Then TPE-DPA-Se was formulated into nanoparticles by nanoprecipitation method with an amphiphilic co-polymer 1,2-distearoyl-sn-glycero-3-phosphoethanolamine- $N$ [methoxy(polyethylene glycol)-2000] (MPEG2000-DSPE) as the doping matrix. TPE-DPA-Se exhibited excellent aqueous diameter stability in nanoparticle-state, as well as impressive luminescence in aqueous system with higher efficiency of up to $16.48 \%$, which are suitable for many fields. To evaluate the biocompatibility of the TPE-DPA-Se NPs, we further carried out 3-(4,5-dimethylthiazol-2-yl)-2,5-diphenyltetrazolium bromide (MTT) cytotoxicity assay. The result indicated their negligible toxicity. Encouraged by the excellent performance of these luminogens in nanoparticle state, a successful 4T1 cells imaging was demonstrated. Next, to set up the tumor-bearing mouse model, the luciferase-expressed 4T1 cancer cells were injected into the healthy mice via intraperitoneal injection. After about 7 days, the abdominal metastatic tumors were formed with many nodules in the abdominal cavity. The fluorescent image can merged with bioluminescence image completely and image-guided tumor resection is verified in this work, particularly for the micro-sized tumor of intraoperative detection.

Keywords aggregation-induced emission; far red/near-infrared; nanoparticles; cell imaging; surgical navigation
\end{abstract}

\section{1 引言}

近年来，苂光生物成像技术由于具有高灵敏度、成
像速度快、实时成像、高生物安全性、检测便捷、成本 低等优势 $[1]$, 在生物成像领域发展迅速 ${ }^{[2-4]}$. 与其它分子

*E-mail: dingd@nankai.edu.cn

Received October 31, 2020; published January 4, 2021.

Supporting information for this article is available free of charge via the Internet at http://sioc-journal.cn.

Project supported by the National Natural Science Foundation of China (Nos. 51961160730, 51873092, 81921004), the National Key R\&D Program of China (Intergovernmental Cooperation Project, 2017YFE0132200), and the Tianjin Science Fund for Distinguished Young Scholars (19JCJQJC61200).

项目受国家自然科学基金(Nos. 51961160730，51873092，81921004)、国家重点研发计划、中国-澳大利亚政府间国际科技创新合作项目 (2017YFE0132200)和天津市杰出青年科学基金(19JCJQJC61200)资助. 
成像技术相比，苂光生物成像技术可以非侵入性实时肿 瘤诊断和成像引导肿瘤切除手术, 辅助外科医生灵敏、 准确地检测和切掉微小的肿瘤, 使肿瘤手术的治疗效果 显著提高 ${ }^{[5]}$. 虽然包括苂光蛋白 ${ }^{[6]}$ 、无机量子点 ${ }^{[7] 、}$ 碳纳

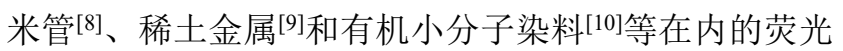
染料迅速发展, 但是各种材料都存在自身局限性, 如苂 光蛋白容易被酶和激光辐照降解; 无机材料生物副作用 大等 ${ }^{[11]}$. 而有机染料因其种类丰富、易修饰、生物相容 性好等优点, 具有良好的应用前景 ${ }^{[12-13]}$. 然而, 在活体 成像应用中，要求染料具有高亮度、高对比度的特性. 因此, 具有组织穿透性高、细胞损伤低、生物自体苂光 干扰小等优势的远红/近红外 $(F R / N I R)$ 发射 $(>650 \mathrm{~nm})$ 的苂光染料，展现出巨大的应用潜力 ${ }^{[14]}$. 目前，一些传 统的有机小分子染料已经被开发出来并用在光介导的 生物医学临床应用中, 如食品药品管理局(FDA)批准的 吲哚菁绿和亚甲基蓝等, 更是凸显了有机小分子染料在 临床转化和实际应用中的潜力 ${ }^{[15]}$.

然而, 传统小分子材料仍存在着诸多问题, 如水体 系中易降解、光稳定性差、斯托克斯位移小、肿瘤积蓄 性差等 ${ }^{[16]}$, 尤其是传统的有机小分子染料往往具有平 面分子结构 ${ }^{[17]}$. 有机小分子染料由于其疏水性往往会 在生物水环境中产生聚集, 聚集体内的分子间相互作用 (如 $\pi-\pi$ 堆积), 极大地阻碍了电子激发态的辐射衰减, 产 生聚集引起淬灭(ACQ)效应，如卟㕲类染料和尼罗红等， 导致荧光染料在生物活体应用中的发射效率、灵敏度和 有效性大幅降低, 限制了实际应用 ${ }^{[18]}$. 因此, 开发出聚 集态下具有高量子产率(QY)、大斯托克斯位移, 稳定性 好的近红外苂光染料仍是一个挑战.

2001 年，唐本忠等 ${ }^{[19]}$ 首次提出的 “聚集诱导发光 (AIE)” 概念, 为解决这一问题开辟了新的途径 ${ }^{[20]}$. 与 ACQ 有机染料相反的是, 聚集诱导发光体(AIEgen)通常 具有周边苯基单元作为分子螺旋桨结构. 因此, AIEgen 在溶解状态下, 激发态电子由于分子内运动(如周边苯 环旋转)而倾向于非辐射耗散, 导致没有荧光或苂光很 弱; 反之, 在聚集状态下, AIEgen 因其具有扭曲的三维 分子结构, 减少了分子间的相互作用; 并且由于分子内 运动/旋转受限(RIM/RIR), 抑制激发态能量的非辐射耗 散, 导致产生强烈的苂光.

AIEgen 的出现解决了传统苂光材料的大部分问题. 尽管许多研究小组已经证明了 AIEgen 在生物成像领域 的独特优势, 但大多数 AIEgen 是疏水的或不稳定的导 致在生物水体系中难以应用. 为了克服这些障碍, 研究 人员广泛采用 PEG- $b$-PPG- $b$-PEG (F127)或 1,2-二硬脂酰 -sn- 甘油-3-磷酸乙醇胺- $N$-[甲氧基-(聚乙二醇)-2000] $\left(\mathrm{MPEG}_{2000}-\mathrm{DSPE}\right)$ 作为掺杂基质共组装制备生物相容性 纳米颗粒(NPs), 该方法可以便捷地提高 AIEgen 的水分 散稳定性和光热稳定性. 同时, 由于纳米颗粒独特的高 渗透/滞留的 “被动” 肿瘤靶向能力, 使负载 AIEgen 的
纳米粒子用于体内癌症诊断成为可能 ${ }^{[21]}$.

要获得具有远红/近红外发射的 AIE 纳米粒子, 研 究人员常采用 $D-\pi-A 、 D-A-D$ 或 A-D-A 方式将电子供体 和受体部分连接成一个分子, 并且所使用的受体部分必 须具有低的能隙. 然而, 这种低能隙有机材料通常表现 出比短发射波长苂光团更低的量子产率. 因此，尽管最 近已有大量新型 AIEgen 被报道 ${ }^{[22-23]}$, 开发出兼具长波 长和高量子产率的染料也具有挑战性.

到目前为止, 苯并塞二唑(BTD)作为一种重要的电 子受体被广泛用于设计高亮度的远红/近红外菼光材料, 但是受 BTD 本身的能隙限制, 得到的材料最大发射波 长较短(约 $600 \mathrm{~nm})^{[24]}$. 因此, 在这项工作中, 我们采用 硫原子下一周期的硒原子, 取代受体结构 BTD 中心的 硫原子, 报道了基于苯并硒二唑(BSD)的 AIEgen 的设计 和合成. BSD 单元表现出比 BTD 单元更强的吸电子能 力, 这种强吸电子能力促进了更红的吸收和发射, 以及 有效的电荷转移. 目前, 含硒元素的 FR/NIR AIE 苂光 染料用于生物成像领域的报道还很少 ${ }^{[25]}$. 为了丰富此 类 AIEgen 家族, 并开发它们在生物领域的应用, 本工 作设计合成并制备了一种高效的 FR/NIR 纳米粒子, 即 TPE-DPA-Se NPs. 其在聚集态(纳米粒子状态)下具有高 量子产率(16.48\%)和相当低的细胞毒性, 体外研究表明, TPE-DPA-Se NPs 可以有效地被细胞内化, 使细胞质着 色. 最后, 研究了 TPE-DPA-Se NPs 在腹腔转移瘤小鼠 模型中的生物成像(图 1). 结果表明, TPE-DPA-Se NPs 在苂光影像引导的手术导航中表现出优异的性能，可以 满足复杂的生物医学应用的要求, 并为 AIE gen 的设计 带来新的见解.

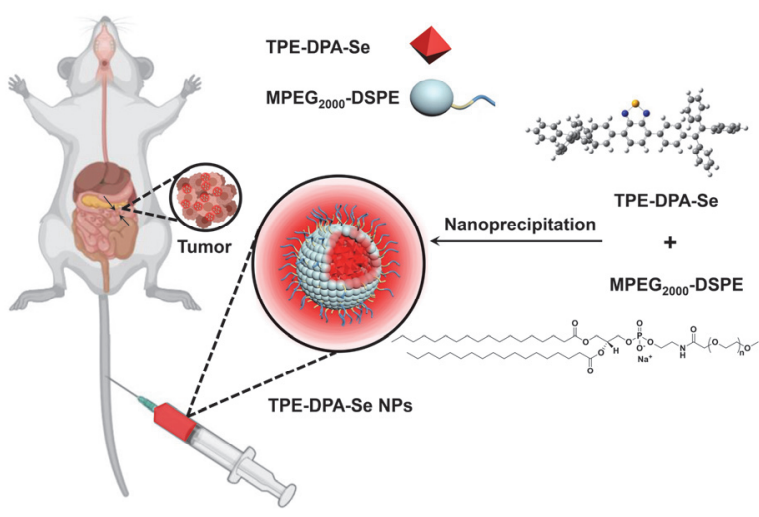

图 1 TPE-DPA-Se-NPs 制备及苂光成像引导肿瘤切除示意图 Figure 1 Schematic illustration of TPE-DPA-Se NPs preparation and fluorescent image-guided tumor resection

\section{2 结果与讨论}

\subsection{TPE-Se、TPA-Se 和 TPE-DPA-Se 的设计与合成}

在这项工作中，基于推拉电子理论，采用 D-A-D 策 略设计和合成了低能隙的 AIEgen. 即选择更低带隙的 苯并硒二唑作为吸电子部分(受体), 在受体两端分别引 
入四苯基乙烯(TPE)、三苯胺(TPA)和四苯基乙烯-二苯胺 (TPE-DPA) 作为供电子部分(供体), 通过调整共轭体系 的相互作用促进分子内电荷转移(ICT), 以此来分别调 整分子红移的最大发射波长和 $\mathrm{AIE}$ 性质. 图 2 描述了化 合物 TPE-Se、TPA-Se 和 TPE-DPA-Se 的合成路线, 所 有中间体、目标化合物均通过 Suzuki-Miyaura 偶联等经 典反应得到. TPE-Se、TPA-Se 和 TPE-DPA-Se 的产率均 在 $75 \%$ 以上, 其化学结构经核磁共振氢谱、碳谱和高分 辨质谱(图 $\mathrm{S} 1 \sim \mathrm{S} 3$ ) 证明了分子结构符合化合物的分子 设计.

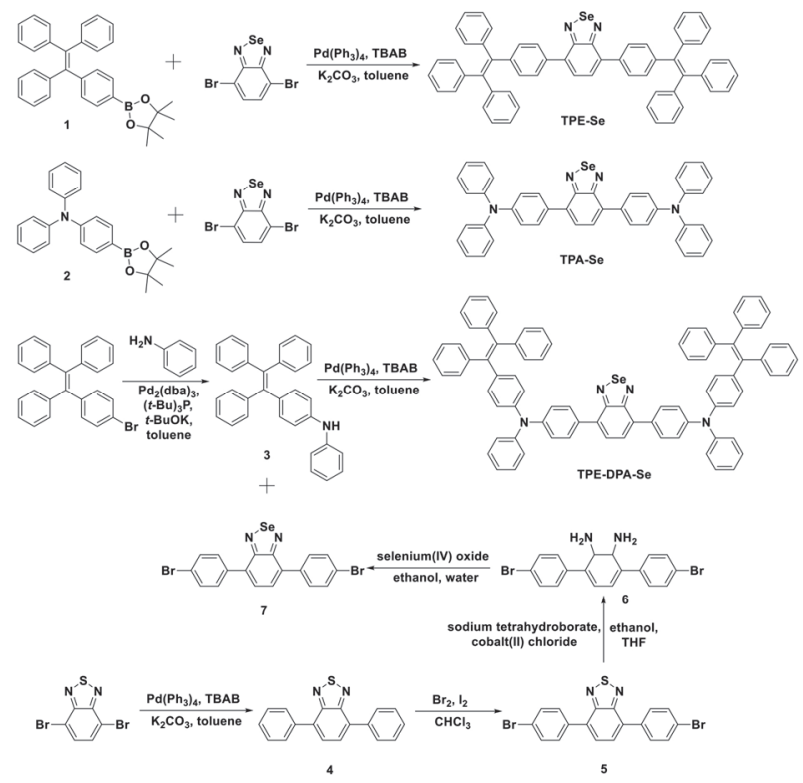

图 2 化合物 TPE-Se、TPA-Se 和 TPE-DPA-Se 的合成路线. Figure 2 Synthetic route for TPE-Se, TPA-Se and TPE-DPA-Se.

\subsection{TPE-Se、TPA-Se 和 TPE-DPA-Se 的光物理性质}

首先, 研究了 TPE-Se、TPA-Se 和 TPE-DPA-Se 的 光学性质包括吸收、发射、 $\mathrm{AIE}$ 性质等. 图 3a 显示了 TPE-Se、TPA-Se 和 TPE-DPA-Se 在四氢呋喃(THF)溶液 中的 UV-Vis 归一化吸收光谱, TPE-Se、TPA-Se 和 TPE-DPA-Se 分子均存在两个主吸收峰, TPE-Se 的峰值 位于 332 和 $422 \mathrm{~nm}$ 附近; TPA-Se 的峰值位于 318 和 480 $\mathrm{nm}$ 左右; 而 TPE-DPA-Se 的峰值位于 333 和 $487 \mathrm{~nm}$ 附 近. 其中波长较短的吸收峰是由于局域 $\pi-\pi$ * 跃迁引起 的, 波长较长的吸收峰则是由于供电子部分和吸电子部 分之间的分子内电荷转移引起(ICT).

与此同时, 如图 $3 b$ 所示, 化合物 TPE-Se、TPA-Se 和 TPE-DPA-Se 在 THF 中的最大发射峰值分别位于 567、647 和 $653 \mathrm{~nm}$. 与化合物 TPE-Se 相比, TPA-Se 和 TPE-DPA-Se 的发射峰红移较大, 分别为 80 和 $86 \mathrm{~nm}$, 表明三苯胺单元和四苯乙烯-二苯胺单元与苯并硒二唑 之间的能量传递强于四苯乙烯单元与苯并硒二唑之间 的能量传递.
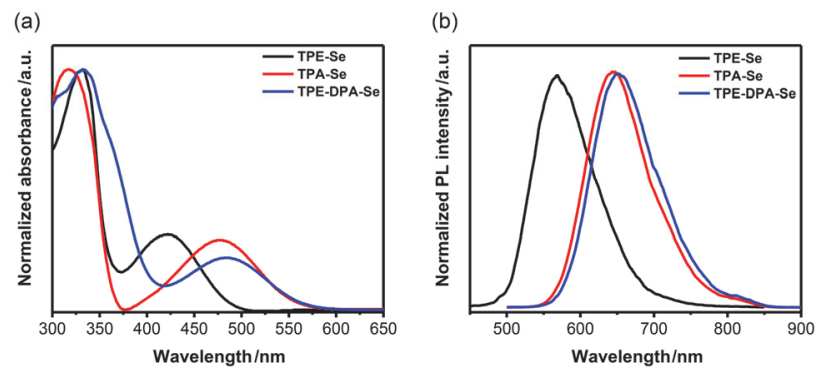

图 3 (a)化合物 TPE-Se、TPA-Se 和 TPE-DPA-Se 在四氢呋喃(THF) 中的归一化 UV-Vis 吸收光谱. (b) 化合物 TPE-Se、TPA-Se 和 TPE-DPA-Se 在四氢呋喃(THF)中的归一化光致发光光谱.

Figure 3 (a) Normalized UV-Vis absorption spectra of compound TPE-Se, TPA-Se, and TPE-DPA-Se in THF. (b) Normalized photoluminescence spectra of compound TPE-Se, TPA-Se, and TPE-DPA-Se in THF.

此外，进一步测试了化合物 TPE-Se、TPA-Se 和 TPE-DPA-Se 在不同 THF/水比例的混合溶液中发射的强 度变化, 以此来验证分子的 AIE 性质. 如图 $4 \mathrm{a} \sim 4 \mathrm{~d}$ 所 示，随着水含量增加，溶剂极性增大，所有化合物苂光 强度降低, 最大发射波长稍有红移, 这一现象可能是由 于分子形成扭曲的分子内电荷转移(twisted intramolecular charge transfer, TICT)态引起的, 分子的发光行为 由 TICT 效应主导. 当水含量超过 $40 \%$ (体积分数)时, 化 (a)
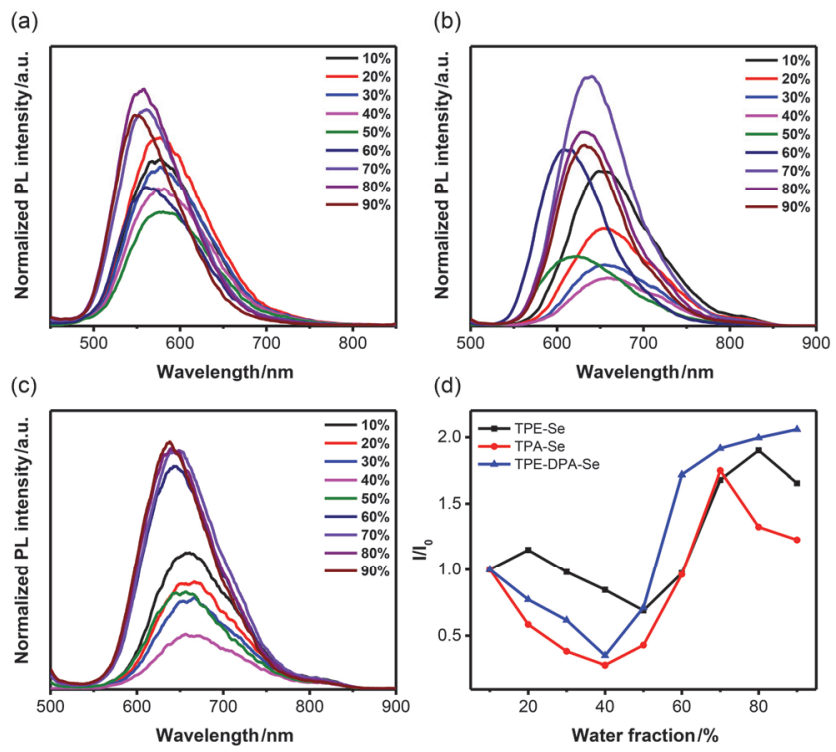

图 4 (a) 化合物 TPE-Se 在不同体积比例下 THF/水混合溶液中的归 一化光致发光光谱 $\left(\lambda_{\mathrm{ex}}=422 \mathrm{~nm}\right)$. (b) 化合物 TPA-Se 在不同体积比例 下 $\mathrm{THF} /$ 水混合溶液中的归一化光致发光光谱 $\left(\lambda_{\mathrm{ex}}=488 \mathrm{~nm}\right.$ ). (c) 化合 物 TPE-DPA-Se 在不同体积比例下 THF/水混合溶液中的归一化光致 发光光谱 $\left(\lambda_{\mathrm{ex}}=488 \mathrm{~nm}\right.$ ). (d) 化合物 TPE-Se、TPA-Se 和 TPE-DPA-Se 的聚集诱导发光曲线 $\left(I_{0}\right.$ 为水体积为 $10 \%$ 时光致发光强度)

Figure 4 (a) Photoluminescence (PL) spectra of TPE-Se in $\mathrm{THF} / \mathrm{H}_{2} \mathrm{O}$ mixtures with different volume fractions of water $\left(\lambda_{\mathrm{ex}}=422 \mathrm{~nm}\right)$. (b) Photoluminescence (PL) spectra of TPA-Se in $\mathrm{THF} / \mathrm{H}_{2} \mathrm{O}$ mixtures with different volume fractions of water $\left(\lambda_{\mathrm{ex}}=488 \mathrm{~nm}\right)$. (c) Photoluminescence (PL) spectra of TPE-DPA-Se in THF/ $\mathrm{H}_{2} \mathrm{O}$ mixtures with different volume fractions of water $\left(\lambda_{\mathrm{ex}}=488 \mathrm{~nm}\right)$. (d) AIE curves of TPE-Se, TPA-Se, and TPE-DPA-Se. $\left(I_{0}\right.$ is the photoluminescence intensity of $10 \%$ water fraction). 
合物 TPA-Se 和 TPE-DPA-Se 的荧光强度逐渐升高，当水 含量超过 $50 \%$ 时，化合物 TPE-Se 的荧光强度也开始升 高, 并伴随着最大发射波长蓝移, 这一现象可能是由于 分子形成聚集体导致的, 此时分子的发光行为转变为由 AIE 性质主导. 同时, 为了确证分子的 TICT 性质, 测试 了化合物在不同极性的溶剂中的光致发光光谱. 如图 S4 所示, 随着溶剂极性的增大, 所有化合物的最大发射 波长逐渐红移. 以上数据表明, 化合物 TPE-Se、TPA-Se 和 TPE-DPA-Se 均兼具 TICT 和 AIE 两种性质. 与此同 时, 图 4d 数据表明, TPE-DPA-Se 分子在水含量为 $90 \%$ 的混合溶液中, 苂光强度继续升高, 展现出最好的 $\mathrm{AIE}$ 性质, 这也为 TPE-DPA-Se 分子进一步在纳米粒子(聚集 态)中的应用奠定了基础.

\subsection{TPE-Se、TPA-Se 和 TPE-DPA-Se 的理论计算}

为了进一步深入研究化合物 TPE-Se、TPA-Se 和 TPE-DPA-Se 的光物理性质, 利用 Gaussian $09 \mathrm{~W}$ 程序在 B3LYP/6-31G**水平上对分子结构进行了密度泛函理论 计算. 图 5a $5 \mathrm{c}$ 分别显示了化合物 TPE-Se、TPA-Se 和 TPE-DPA-Se 的优化结构、最高占据分子轨道(HOMO) 和最低未占据分子轨道(LUMO)分布情况. 这些化合物 的优化几何结构表明, 它们都表现出相当扭曲的三维几 何结构, 这将阻碍聚集状态下的分子堆积(如 $\pi-\pi$ 堆积), 有利于激发态能量的辐射耗散 ${ }^{[26]}$. 图中结果表明, 化合 物的扭曲程度从高到低依次排序为: TPE-DPA-Se、 TPE-Se 和 TPA-Se. 这与光物理性质的实验结果一致, TPE-DPA-Se 展现出最好的 AIE 性质. 图 5 的计算结果 还显示 TPE-Se、TPA-Se 和 TPE-DPA-Se 的 LUMO 主要 集中分布在苯并硒二唑的吸电子部分. 与此同时, TPE-Se 和 TPA-Se 的 HOMO 沿着分子骨架均匀分布, 而 TPE-DPA-Se 的 HOMO 主要集中在三苯胺部分, 这一结 果更确证了 ICT 效应的存在. 化合物 TPE-Se、TPA-Se 和 TPE-DPA-Se 的 HOMO/LUMO 能级分别为 $-5.15 \mathrm{eV} /$ $-2.45 \mathrm{eV} 、-4.79 \mathrm{eV} /-2.38 \mathrm{eV}$ 和 $-4.70 \mathrm{eV} /-2.38 \mathrm{eV}$. 此外, 我们计算得到 TPE-Se、TPA-Se 和 TPE-DPA-Se 的能隙分别为 $2.70 、 2.41$ 和 $2.32 \mathrm{eV}$, 说明供电子部分越 强, 能隙越小, 发射波长红移, 与其光物理性质的结果 一致.

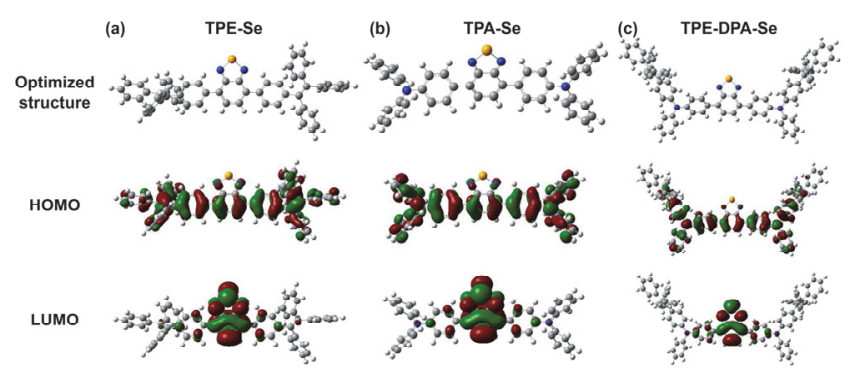

图 5 化合物 TPE-Se (a)、TPA-Se (b)和 TPE-DPA-Se (c)的优化结构、 最高占据分子轨道(HOMO) 和最低未占据分子轨道 (LUMO)的分布 Figure 5 The optimized structure, HOMO and LUMO orbital distributions of TPE-Se (a), TPA-Se (b), and TPE-DPA-Se (c)

\section{4 纳米粒子的制备、表征以及光物理性质}

为了使 AIEgen 能够被应用于生物环境水体系, 以 一种两亲性共聚物 $\mathrm{MPEG}_{2000}-\mathrm{DSPE}$ 为掺杂基质, 采用 超声共沉淀的方法制备纳米粒子 [27]. 其方法是将 AIEgen 和 $M_{2}$ EG $_{2000}-D S P E$ 溶解在纯 THF 溶液中, 在超 声条件下向水中缓慢滴加，在超声作用和亲疏水相互作 用下制备成负载 AIEgen 的纳米粒子, 然后用持续的氮 气吹拂浓缩至指定浓度. 疏水的 AIEgen 和 $\mathrm{MPEG}_{2000}-\mathrm{DSPE}$ 的疏水部分相互作用，形成纳米粒子的 内核，而亲水性的聚乙二醇链则分散在粒子表面，使粒 子均匀而稳定的分散在水体系中, 防止沉淀. 动态光散 射(DLS)数据显示, TPE-Se、TPA-Se 和 TPE-DPA-Se 掺 杂的 AIE NPs 的平均流体力学直径(图 $6 a \sim 6 c$ )分别为 109、120 和 $113 \mathrm{~nm}$. 这使得纳米粒子通过渗透和保留 (EPR)效应具有适用于肿瘤聚集的被动靶向能力 ${ }^{[21]}$. 此 外，据文献报道，这种尺寸处于 $100 \mathrm{~nm}$ 附近的纳米粒子 有利于体内长时间血液循环和良好的高渗透长滞留 (EPR) 效应 ${ }^{[28]}$. 此外, 我们还利用透射电子显微镜 (TEM)对这三个 AIE NPs 的形貌进行了表征. 如图 6a $6 \mathrm{c}$ (插图)所示, 这三个 AIE NPs 均表现出类球形的形状. 同时, 结果表明(图 6d), 在 $4{ }^{\circ} \mathrm{C}$ 条件下保存 4 周后, 溶 液的直径没有变化, 也没有观察到沉淀和聚集现象, 表 明纳米颗粒具有良好的胶体稳定性.
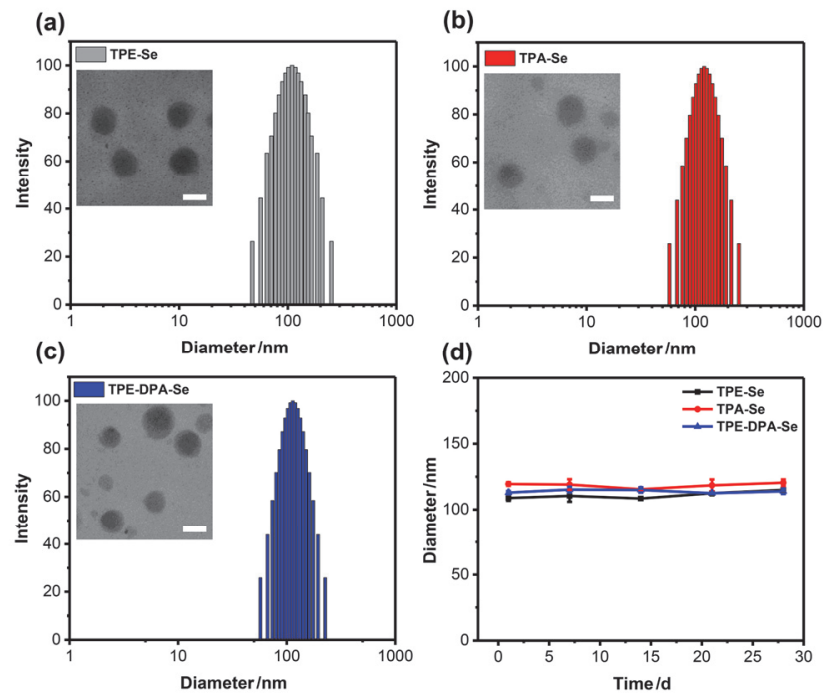

图 6 TPE-Se NPs (a)、TPA-Se NPs (b) 和 TPE-DPA-Se NPs (c)的动态 光散射直径 (DLS). 插图: TPE-Se NPs (a), TPA-Se NPs(b) 和 TPE-DPA-Se NPs (c)的透射电子显微镜图(TEM). (d) 纳米粒子在 $4{ }^{\circ} \mathrm{C}$ 条件下存储 $1,7,14,21$ 和 $28 \mathrm{~d}$ 后的粒径

Figure 6 The DLS distributions of TPE-Se NPs (a), TPA-Se NPs (b), and TPE-DPA-Se NPs (c). Inset: the TEM images of TPE-Se NPs (a), TPA-Se NPs (b), and TPE-DPA-Se NPs (c). (d) The diameter of nanoparticles after storage at $4{ }^{\circ} \mathrm{C}$ for $1,7,14,21$ and $28 \mathrm{~d}$

进一步地, 我们研究了 AIE NPs 在水介质中的光物 理性质. 由图 7a 的照片可以观察到, AIE NPs 在 $365 \mathrm{~nm}$ 的紫外灯照射下可以发出明亮的苂光, 并且 TPA-Se 
NPs 和 TPE-DPA-Se NPs 可以发出近红外的明亮的红色 菼光. 图 $7 \mathrm{~b} \sim 7 \mathrm{~d}$ 显示了三种纳米粒子在水中的归一化 UV-Vis 吸收光谱和归一化苂光光谱. 与在 THF 中的 TPE-Se、TPA-Se 和 TPE-DPA-Se 溶液相比, 这三种纳米 粒子的 UV-Vis 吸收的峰形与主峰位置都没有明显变化, 而 TPE-Se NPs、TPA-Se NPs 和 TPE-DPA-Se NPs 的发 射峰的峰值则均表现出轻微的蓝移, 分别位于 551, 626 和 $642 \mathrm{~nm}$, 这可能是由于分子形成了聚集体导致的. 值 得注意的是, 对比 TPE-Se 溶液和 TPE-Se NPs, TPE-DPA-Se 溶液和 TPE-DPA-Se NPs, 纳米粒子的最大 发射峰相较于稀溶液中蓝移约 $10 \mathrm{~nm}$, 而相同情况下, TPA-Se NPs 相较于 TPA-Se 稀溶液蓝移约 $20 \mathrm{~nm}$. 这一 结果从另一方面证明了四苯乙烯部分比三苯胺部分更 有效地阻碍在聚集状态下的分子堆积. 此外, 采用校准 积分球的方法测量了 TPE-Se、TPA-Se 和 TPE-DPA-Se 掺杂的 AIE NPs 在水中的量子产率(QY)的值分别为 $12.01 \%$ 、13.88\% 和 16.48\%. TPE-DPA-Se NPs 的量子产 率高于 TPA-Se NPs 的这一现象不同于以往的大多数结 果, 即非辐射衰减通常随着带隙的减小而增加. 这可能 是由于在聚集状态下分子运动受阻, 扭曲的分子结构抑 制非辐射衰减占据主要地位, 这与我们的实验结果一致 (图 4d), 而且, 这在文献中也得到了很好的印证 ${ }^{[24]}$. (a)

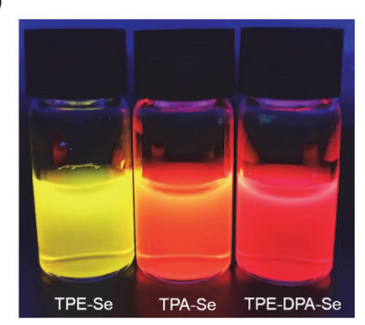

(c)

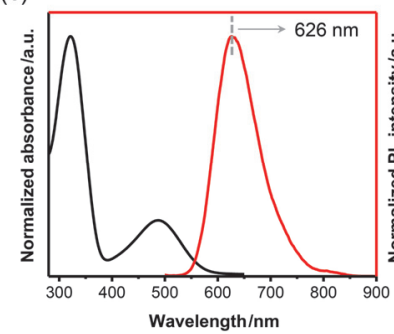

(b)

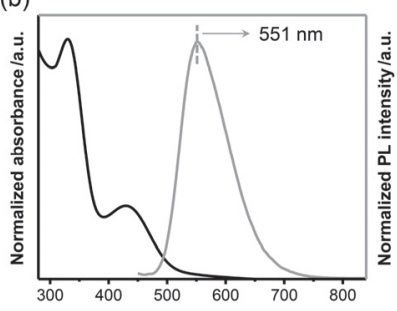

(d)

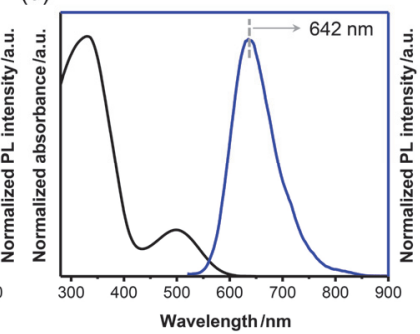

图 7 (a) TPE-Se NPs、TPA-Se NPs 和 TPE-DPA-Se NPs 在 $365 \mathrm{~nm}$ 紫 外光照射下的荧光照片. TPE-Se NPs (b)、TPA-Se NPs (c) 与 TPE-DPA-Se NPs (d) 的归一化 UV-Vis 吸收光谱和归一化光致发光光 谱

Figure 7 (a) The fluorescent image of TPE-Se NPs, TPA-Se NPs, and TPE-DPA-Se NPs under illumination of $365 \mathrm{~nm}$ UV light. The normalized UV-Vis absorption and the normalized photoluminescence spectra of TPE-Se NPs (b), TPA-Se NPs (c), and TPE-DPA-Se NPs (d)

\subsection{TPE-DPA-Se NPs 的细胞毒性试验和细胞成像}

由于 TPE-DPA-Se NPs 在上述实验结果中表现出最 近红外的最大吸收和苂光发射, 以及最好的 AIE 性质和 最高的量子产率, 有利于进一步的体外、体内生物成像
研究, 我们选取 TPE-DPA-Se NPs 进行了下一步的生物 应用研究. 我们用小鼠乳腺癌细胞(4T1 细胞)进行 3-(4,5-二甲基噻唑-2-基)-2,5-二苯基四唑溴化铵(MTT) 细胞毒性评价. 图 S5 为不同浓度的 TPE-DPA-Se NPs 与 $4 \mathrm{~T} 1$ 细胞共孵育 $12 \mathrm{~h}$ 的细胞活性. 结果表明, 在最大为 $200 \mu \mathrm{mol} / \mathrm{L}$ 的浓度下，细胞活性仍保持在 $90 \%$ 以上，表 明 TPE-DPA-Se NPs 基本无毒且生物相容性非常好.

在验证了 TPE-DPA-Se NPs 的细胞安全性之后, 进 行了体外细胞成像研究. 用浓度为 $20 \mu \mathrm{mol} / \mathrm{L}$ 的纳米粒 子与 $4 \mathrm{~T} 1$ 细胞在 $37{ }^{\circ} \mathrm{C}$ 条件下共孵育 $4 \mathrm{~h}$, 然后加入 4',6-diamidino-2-phenylindole (DAPI)培养 5 10 min, 使 细胞核着色. 随后, 使用共聚焦激光扫描显微镜 (CLSM) 捕捉细胞内苂光图像, 以此确定 TPE-DPA-Se NPs 作为生物成像染料的潜力. 首先用 CLSM 采集了 4T1 细胞的明场照片(图 8a), 随后分别在 $405 \mathrm{~nm}$ (DAPI) 和 $488 \mathrm{~nm}$ (TPE-DPA-Se NPs) 的激发光源下, 捕获 DAPI (图 8b) 和 TPE-DPA-Se NPs (图 8c)的苂光信号, 再叠加 图 $8 \mathrm{~b}$ 和图 $8 \mathrm{c}$ 获得图 $8 \mathrm{~d}$. 结果显示, $4 \mathrm{~T} 1$ 细胞的细胞质中 有强烈的红色荧光, 可以很好地与 DAPI 着色的细胞核 共同定位细胞，证明了 TPE-DPA-Se NPs 可以被 4T1 细 胞有效地内化并滞留在细胞质中.

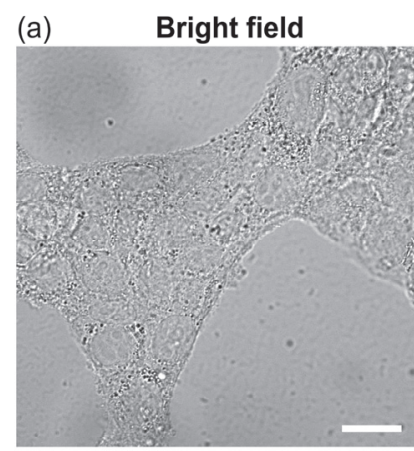

(c)

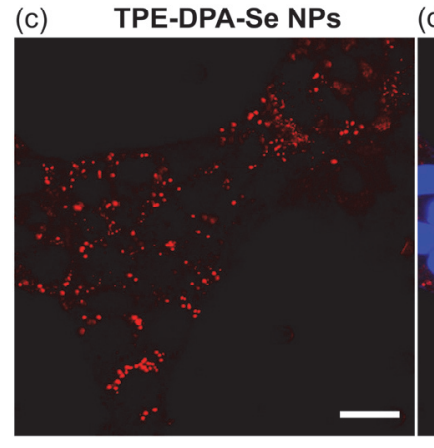

(b)
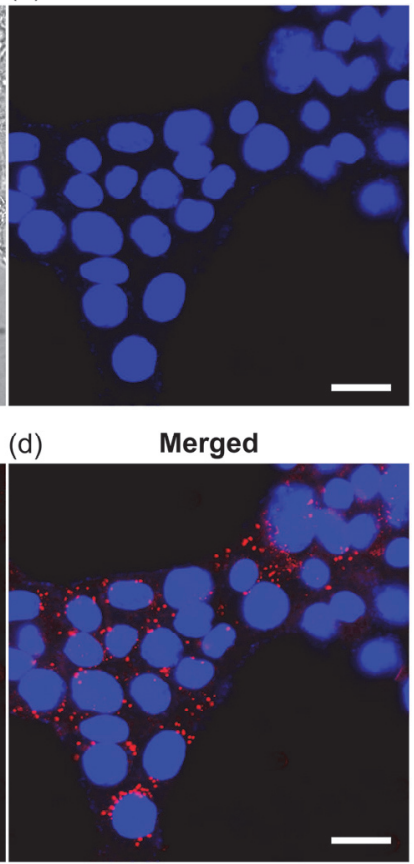

图 8 共聚焦激光扫描显微镜(CLSM)观察 $4 \mathrm{~T} 1$ 乳腺癌细胞与 TPE-DPA-Se-NPs 共孵育 $4 \mathrm{~h}$ 后的图像, 细胞核用 DAPI(蓝色)染色. 比 例尺为 $20 \mu \mathrm{m}$.

Figure 8 Confocal laser scanning microscope (CLSM) images of 4T1 breast cancer cells after incubation with TPE-DPA-Se NPs for $4 \mathrm{~h}$. The cell nuclei were stained with DAPI (blue). Scale bars indicate $20 \mu \mathrm{m}$.

\subsection{TPE-DPA-Se NPs 的荧光影像引导肿瘤切除}

在临床外科肿瘤学中, 术中影像通过对肿瘤结节的 精确辨别和切除, 对提高手术效果具有相当大的作用. 
近红外苂光成像技术是一种很有前景的高灵敏度肿瘤 边缘识别技术, 目前已被多篇报道证实 ${ }^{[28-30]}$. 乳腺癌是 一种极其普遍的疾病, 据估计全球有八分之一的女性在 一生中会患有这种疾病, 是一个严重的公共卫生问题. 并且, 乳腺癌的腹腔转移是导致总生存率低的主要因素 之一, 由于腹膜腔内分散着许多微小的肿瘤结节, 因此 术前评估和术中影像极具挑战性. 这些难以发现的微小 肿瘤结节是肿瘤复发的主要原因.

因此, 我们建立了 $4 \mathrm{~T} 1$ 癌细胞腹膜转移的小鼠模 型, 以评估 TPE-DPA-Se NPs 辅助微小肿瘤结节的外科 鉴别和切除. 为了使肿瘤的分布可以被监测, 我们选择 了苂光素酶表达的 4T1 肿瘤, 这些肿瘤在注射荧光素后 显示出生物发光. 将 TPE-DPA-Se NPs 尾静脉注射入小 鼠体内循环 $12 \mathrm{~h}$ 后, TPE-DPA-Se NPs 的苂光与苂光素 的生物发光信号在腹腔内完全重合 (图 9a), 证明了 TPE-DPA-Se NPs 可以通过 EPR 效应对肿瘤的准确诊断.

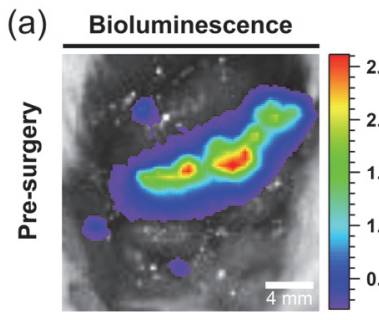

(b) Post-unguided surgery

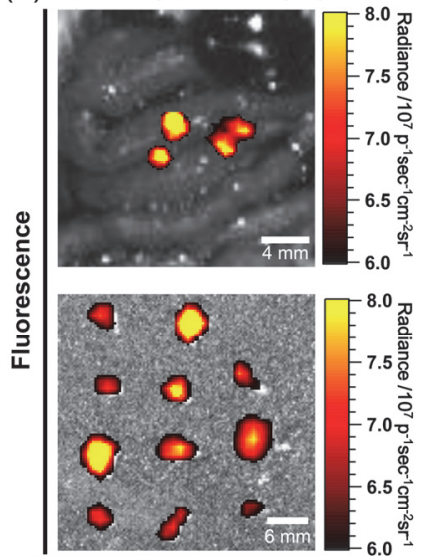

(c)

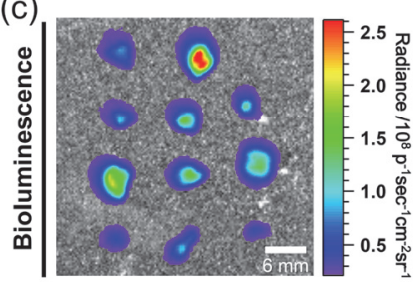

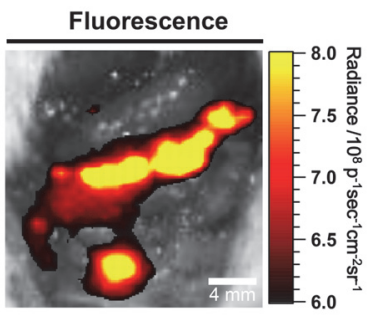

Post-image-guided surgery
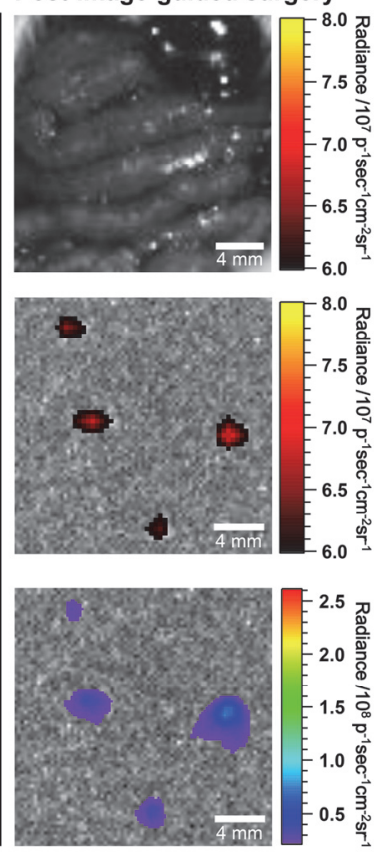

图 9 (a) 肿瘤切除前小鼠腹腔的生物发光和苂光显像图. (b) 苂光影 像引导切除前后的腹腔苂光图像和肿瘤结节苂光显像. (c) 未引导组 和 TPE-DPA-Se NPs 引导组切除的肿瘤结节的生物发光图像.

Figure 9 (a) Bioluminescence and fluorescence imaging of the abdominal cavity before tumor resection. (b) Fluorescence imaging of the abdominal cavity before and after image-guided tumor resection, and the fluorescence imaging of resected nodules. (c) Bioluminescence imaging of resected nodules of unguided and TPE-DPA-Se NPs-guided groups.
在临床上, 外科医生主要依靠肉眼分辨哪些组织需要切 除和保存. 虽然相对较大 $(>1 \mathrm{~mm})$ 的肿瘤已被外科医生 (天津市第一中心医院)在肉眼分辨条件下切除, 但仍有 一些微小的肿瘤结节残留，难以被识别. 因此，在近红 外苂光的引导下进行了二次手术切除，观察到肿瘤被基 本切除干净(图 9b). 所有切除的肿瘤结节的生物发光信 号与荧光信号重合, 证实切除的组织为肿瘤(图 9c). 此 外，我们还对小鼠体内 TPE-DPA-Se NPs 的生物分布进 行了研究, 结果显示(图 S6), 静脉注射 TPE-DPA-Se NPs 后，由于众所周知的网状内皮系统(RES)摄取，在注射 后的 $12 \mathrm{~h}$ 内, 在肝脏和脾脏中观察到强烈的荧光信号. 更进一步地, 我们进行了苏木精和伊红(H\&E)组织学分 析, 证实 TPE-DPA-Se NPs 对主要器官无明显损害(图 S7), 并且切除的微小组织确实为肿瘤(图 S8). 这些结果 表明, 用 TPE-DPA-Se NPs 准确识别微小肿瘤结节可以 提高肿瘤手术疗效, 大大降低肿瘤原位复发的风险.

\section{3 结论}

综上所述，我们采用引入扭曲基团(TPE)提升分子 聚集态发光性质的策略, 合成了三种具有 D-A-D 结构 的基于苯并硒二唑电子受体的分子, 其中, TPE-DPA-Se 分子表现出最好的聚集诱导发光性质.随后，利用纳米 沉淀法，将 $\mathrm{MPEG}_{2000}-\mathrm{DSPE}$ 作为掺杂基质，制备了粒径 在 $100 \mathrm{~nm}$ 尺寸附近的纳米粒子. 同样, 兼具扭曲三维构 型(TPE)和强供电子部分(DPA)的 TPE-DPA-Se 展现出最 长波长的吸收/发射峰 $(487 \mathrm{~nm} / 653 \mathrm{~nm})$ 和在纳米粒子状 态下最高的绝对量子产率 $(16.48 \%)$. 体外研究表明, TPE-DPA-Se NPs 基本无毒、生物相容性良好，并且可以 有效地被细胞内化, 使细胞质着色. 进一步的体内研究 表明，静脉注射明亮的 TPE-DPA-Se NPs，能够在手术 中清晰地显示腹膜转移瘤及与正常组织边界, 指导医生 切除微小肿瘤. 在临床应用中, 有望使病人最大程度的 保留正常组织，提升预后效果，降低病人癌症复发风险. 本研究也将激励人们进一步开发用于复杂生物医学应 用的新型 AIE 分子和探针.

\section{References}

[1] (a) Antaris, A. L.; Chen, H.; Cheng, K.; Sun, Y.; Hong, G.; Qu, C.; Diao, S.; Deng, Z.; Hu, X.; Zhang, B.; Zhang, X.; Yaghi, O. K.; Alamparambil, Z. R.; Hong, X.; Cheng, Z.; Dai, H. Nat. Mater. 2016, 15, 235. (b) Qi, J.; Chen, C.; Zhang, X.; Hu, X.; Ji, S.; Kwok, R. T. K.; Lam, J. W. Y.; Ding, D.; Tang, B. Z. Nat. Commun. 2018, 9 , 1848. (c) Koch, M.; Symvoulidis, P.; Ntziachristos, V. Nat. Photonics 2018, 12, 505. (d) Jiang, Y.; Pu, K. Acc. Chem. Res. 2018, 51 , 1840. (e) Gao, M.; Yu, F.; Lv, C.; Choo, J.; Chen, L. Chem. Soc. Rev. 2017, 46, 2237. (f) Zheng, X. C.; Mao, H.; Huo, D.; Wu, W.; Liu, B. R.; Jiang, X. Q. Nat. Biomed. Eng. 2017, 1, 0057.

[2] Yang, M.; Xia, L. L.; Zhou, X. Q.; Jia, C. L.; Ji, M.; Wang, P. Chin. J. Org. Chem. 2020, 40, 2888 (in Chinese). (杨敏, 夏丽丽, 周小琴, 贾程利, 吉民, 王鹏, 有机化学, 2020, 40, 2888.)

[3] Huang, C. B.; Chen, H.; Li, F. Q.; An, S. Y. Chin. J. Org. Chem. 2019, 39, 2467 (in Chinese). (黄池宝, 陈会, 李福琴, 安思雅, 有 机化学, 2019, 39, 2467.)

[4] Yan, T.; Liu, Z. H.; Song, X. Y.; Zhang, S. S. Acta Chim. Sinica 
2020, 78, 657 (in Chinese). (问涛, 刘振华, 宋昕玥, 张书圣, 化学 学报, 2020, 78, 657.)

[5] (a) Vahrmeijer, A. L.; Hutteman, M.; van der Vorst, J. R.; van de Velde, C. J. H.; Frangioni, J. V. Nat. Rev. Clin. Oncol. 2013, 10, 507. (b) Colby, A. H.; Berry, S. M.; Moran, A. M.; Pasion, K. A.; Liu, R.; Colson, Y. L.; Ruiz-Opazo, N.; Grinstaff, M. W.; Herrera, V. L. M. ACS Nano 2017, 11, 1466. (c) Choi, H. S.; Gibbs, S. L.; Lee, J. H.; Kim, S. H.; Ashitate, Y.; Liu, F.; Hyun, H.; Park, G.; Xie, Y.; Bae, S.; Henary, M.; Frangioni, J. V. Nat. Biotechnol. 2013, 31, 148.

[6] Zimmer, M. Chem. Rev. 2002, 102, 759.

[7] Wegner K. D.; Hildebrandt, N. Chem. Soc. Rev. 2015, 44, 4792

[8] Ghosh, D.; Bagley, A. F.; Na, Y. J.; Birrer, M. J.; Bhatia, S. N.; Belcher, A. M. Proc. Natl. Acad. Sci. U. S. A. 2014, 111, 13948.

[9] Xiong, L.; Fan, Y.; Zhang, F. Acta Chim. Sinica 2019, 77, 1239 (in Chinese). (能麟, 凡勇, 张凡, 化学学报, 2019, 77, 1239.)

[10] Kobayashi, H.; Ogawa, M.; Alford, R.; Choyke, P. L.; Urano, Y. Chem. Rev. 2010, 110, 2620.

[11] Derfus, A. M.; Chan, W. C. W.; Bhatia, S. N. Nano Lett. 2004, 4, 11.

[12] (a) He, X. X.; Wang, K. M.; Cheng, Z. Interdiscip. Rev. Nanomed. Nanobiotechnol. 2010, 2, 349. (b) Larush, L.; Magdassi, S. Nanomedicine 2011, 6, 233. (c) Hilderbrand, S. A.; Weissleder, R. Curr. Opin. Chem. Biol. 2010, 14, 71 .

[13] Zhang, L; Zhao, W. L.; Li, M; Lv, H. Y; Chen, C. F. Acta Chim. Sinica 2020, 78, 1030 (in Chinese). (张亮，赵文龙，李猛，吕海燕， 陈传峰, 化学学报, 2020, 78, 1030.)

[14] (a) Gao, G. B.; Gong, D. J.; Zhang, M. X.; Sun, T. L. Acta Chim Sinica 2016, 74, 363 (in Chinese). (高冠斌, 龚德君, 张明曦, 孙涛 垒, 化学学报, 2016, 74, 363.) (b) Ji, G.; Yan, L. L.; Wang, H.; Ma, L.; Xu, B.; Tian, W. J. Acta Chim. Sinica 2016, 74, 917 (in Chinese). (纪光, 问路林, 王慧, 马莲, 徐斌, 田文晶, 化学学报, 2016, 74, 917).

[15] (a) Chi, C.; Du, Y.; Ye, J.; Kou, D.; Qiu, J.; Wang, J.; Tian, J.; Chen, X. Theranostics 2014, 4, 1072. (b) Olson, M. T.; Ly, Q. P.; Mohs, A. M. Mol. Imaging Biol. 2018, 4, 1072. (c) Manen, L. V.; Handgraaf, H. J. M.; Diana, M.; Dijkstra, J.; Ishizawa, T.; Vahrmeijer, A. L.; Mieog, J. S. D. Surg, J. Oncol. 2018, 118, 283.

[16] (a) Qi, J.; Fang, Y.; Kwok, R. T. K.; Zhang, X.; Hu, X.; Lam, J. W. Y.; Ding, D.; Tang, B. Z. ACS Nano 2017, 11, 7177. (b) Li, K.; Ding, D.; Zhao, Q.; Sun, J.; Tang, B. Z.; Liu, B. Sci. China Chem. 2013, 56, 1228. (c) Liu, Z.; Chen, K.; Davis, C.; Sherlock, S.; Cao, Q.; Chen, X.; Dai, H. Cancer Res. 2008, 68, 6652. (d) Derfus, A. M.; Chan, W. C. W.; Bhatia, S. N. Nano Lett. 2004, 4, 11.

[17] Hong, Y.; Lam, J. W. Y.; Tang, B. Z. Chem. Commun. 2009, 4332.

[18] (a) Sakakibara, Y.; Okutsu, S.; Enokida T.; Tani, T. Appl. Phys. Lett. 1999, 74, 2587. (b) Chiang, C. L.; Wu, M. F.; Dai, D. C.; Wen, Y. S.; Wang J. K.; Chen, C. T. Adv. Funct. Mater. 2005, 15, 231.
[19] (a) Mei, J.; Leung, L. C.; Kwok, T. K.; Lam, W. Y.; Tang, B. Z. Chem. Rev. 2015, 115, 11718. (b) Xia, Z. Q.; Shao, A. D.; Li, Q.; Zhu, S. Q.; Zhu, W. H. Acta Chim. Sinica 2016, 74, 351 (in Chinese). (夏志清, 邵安东, 李强, 朱世琴, 朱为宏, 化学学报, 2016, 74, 351.)

[20] (a) Gao, H.; Zhang, X.; Chen, C.; Li, K.; Ding, D. Adv. Biosyst. 2018, 2, 1800074. (b) Qin, A.; Tang, B. Z. Sci. China Chem. 2018 61, 879. (c) Qi, J.; Chen, C.; Ding, D.; Tang, B. Z. Adv. Healthcare Mater. 2018, 7, 1800477. (d) Ding, D.; Li, K.; Liu, B.; Tang, B. Z. Acc. Chem. Res. 2013, 46, 2441.

[21] (a) Lu, Y.; Aimetti, A. A.; Langer, R.; Gu, Z. Nat. Rev. Mater. 2017, 2, 16075. (b) Farokhzad, O. C.; Langer, R. ACS Nano 2009, 3, 16 (c) Xia, L.; Cheng, Z.; Zhu, H.; Yang, Z. Acta Chim. Sinica 2019 77, 172 (in Chinese). (夏雷, 程震, 朱华, 杨志, 化学学报, 2019 , $77,172$.

[22] (a) Wang, J.; Wu, Y. L.; Sun, L. H.; Zeng, F.; Wu, S. Z. Acta Chim Sinica 2016, 74, 910 (in Chinese). (王俊, 武英龙, 孙立和, 曾钫, 吴水珠，化学学报, 2016, 74, 910.) (b) Sang, R. Y.; Xu, X. P.; Wang, Q.; Fan, Q. L.; Huang, W. Acta Chim. Sinica 2020, 78, 901 (in Chinese). (桑若愚, 许兴鹏, 王其, 范曲立, 黄维, 化学学报, 2020, 78, 901).

[23] Guan, X. L.; Wang, L.; Li, Z. F.; Liu, M. N.; Wang, K. L.; Lin, B.; Yang, X. Q; Lai, S. J.; Lei, Z. Q. Acta Chim. Sinica 2019, 77, 1036 (in Chinese). (关晓琳, 王林, 李志飞, 刘美娜, 王凯龙, 林斌, 杨 学琴, 来守军, 雷自强, 化学学报, 2019, 77, 1036.)

[24] Qin, W.; Li, Kai.; Feng, G. X.; Li, M.; Yang, Z. Y.; Liu, B.; Tang, B. Z. Adv. Funct. Mater. 2014, 24, 635.

[25] Qin, W.; Alifu, N.; Cai, Y. J.; Lam, J. W. Y.; He, X. W.; Su, H. F.; Zhang, P. F.; Qian, J.; Tang, B. Z. Chem. Commun. 2019, 55, 5615.

[26] Qi, J.; Duan, X. J.; Cai, Y. J.; Jia, S. R.; Chen, C.; Zhao, Z.; Li, Y.; Peng, H. Q.; Kwok, R. T. K.; Lam, J. W. Y.; Ding, D.; Tang, B. Z. Chem. Sci. 2020, 11, 8438.

[27] Zhen, X.; Tao, Y.; An, Z. F.; Chen, P.; Xu, C. J.; Chen, R. F.; Huang, W.; Pu, K. Y. Adv. Mater. 2017, 29, 1606665.

[28] Pandit, S.; Dutta, D.; Nie, S. Nat. Mater. 2020, 19, 478

[29] (a) Sun, Y.; Ding, M.; Zeng, X.; Xiao, Y.; Wu, H.; Zhou, H.; Ding, B.; Qu, C.; Hou, W.; Er-Bu, A.; Zhang, Y.; Cheng, Z.; Hong, X. Chem. Sci. 2017, 8, 3489. (b) Vahrmeijer, A. L.; Hutteman, M.; van der Vorst, J. R.; van de Velde, C. J.; Frangioni, J. V. Nat. Rev. Clin. Oncol. 2013, 10, 507.

[30] Luo, X. R.; Chen, M. W.; Yang, Q. L. Acta Chim. Sinica 2020, 78, 373 (in Chinese). (罗兴芯荵，陈敏文，杨晴来，化学学报，2020, 78, 373.)

(Cheng, B. 